\title{
Dependence of hadronic interaction models in atmospheric electric field simulations for GRAPES-3
}

\author{
B. Hariharan ${ }^{* 1,2,3}$, S. Ahmad ${ }^{1,4}$, M. Chakraborty ${ }^{1,2}$, A. Chandra ${ }^{1,4}$, S.R. Dugad ${ }^{1,2}$, \\ S.K. Gupta ${ }^{1,2}$, Y. Hayashi ${ }^{1,5}$, S.S.R. Inbanathan ${ }^{1,3}$, P. Jagadeesan ${ }^{1,2}$, A. Jain ${ }^{1,2}$, \\ P. Jain ${ }^{1,6}$, V.B. Jhansi ${ }^{1,2}$, S. Kawakami ${ }^{1,5}$, H. Kojima ${ }^{1,7}$, S. Mahapatra ${ }^{1,8}$, \\ P.K. Mohanty ${ }^{1,2}$, S.D. Morris ${ }^{1,2}$, Y. Muraki ${ }^{1,9}$, P.K. Nayak ${ }^{1,2}$, A. Oshima ${ }^{1,7}$, \\ D. Pattanaik ${ }^{1,2}$, P.S. Rakshe ${ }^{1,2}$, K. Ramesh ${ }^{1,2}$, B.S. Rao ${ }^{1,2}$, L.V. Reddy ${ }^{1,2}$, S. Sharma ${ }^{1,2}$, \\ S. Shibata ${ }^{1,7}$, K. Tanaka ${ }^{1,10}$, F. Varsi ${ }^{1,6}$, M. Zuberi ${ }^{1,2}$ \\ ${ }^{1}$ The GRAPES-3 Experiment, Cosmic Ray Laboratory, Raj Bhavan, Ooty 643001, India \\ ${ }^{2}$ Tata Institute of Fundamental Research, Mumbai 400005, India \\ ${ }^{3}$ The American College, Madurai 625002, India \\ ${ }^{4}$ Aligarh Muslim University, Aligarh 202002, India \\ ${ }^{5}$ Graduate School of Science, Osaka City University, Osaka 558-8585, Japan \\ ${ }^{6}$ Indian Institute of Technology Kanpur, Kanpur 208016, India \\ ${ }^{7}$ College of Engineering, Chubu University, Kasugai, Aichi 487-8501, Japan \\ ${ }^{8}$ Utkal University, Bhuvaneshwar 751004, India \\ ${ }^{9}$ Institute for Space-Earth Environmental Research, Nagoya University, Nagoya 464-8601, Japan \\ ${ }^{10}$ Graduate School of Information Sciences, Hiroshima City University, Hiroshima 731-3194, \\ Japan \\ E-Mail: 89hariharanegmail.com
}

\begin{abstract}
The thunderstorm events observed by GRAPES-3 muon telescope can be studied with aid of Monte Carlo simulations. One such event observed on 1 December 2014 was analysed and its electrical properties of thundercloud were derived using muon imaging. This recent result showed production of Giga-Volt potential in thunderclouds, possibly responsible for production of $100 \mathrm{MeV} \gamma$-rays in terrestrial $\gamma$-ray flashes. However, these properties derived from simulations rely on choice of low and high energy hadronic interaction models used in CORSIKA. So, the derived properties are model dependent which makes this study very important. The comparison of various combinations using low and high energy hadronic models and its impact on electric potential estimation will be discussed in this work.
\end{abstract}

36th International Cosmic Ray Conference -ICRC2019-

July 24th - August 1st, 2019

Madison, WI, U.S.A.

${ }^{*}$ Speaker. 


\section{Introduction}

The GRAPES-3 experiment located at Ooty, India $\left(11.4^{\circ} \mathrm{N}, 76.7^{\circ} \mathrm{E}, 2200 \mathrm{~m}\right.$ amsl) recently reported a result on measurement of electrical properties of one of the largest thunderstorm event recorded by GRAPES-3 muon telescope (G3MT) using change in muon intensity $\left(\Delta \mathrm{I}_{\mu}\right)$ above $\mathrm{GeV}$ threshold [1]. This thunderstorm event which was recorded on 1 December 2014 lasted for 18 minutes. During peak activity, the $\Delta \mathrm{I}_{\mu}$ dropped as low as $2 \%$. By combining electric field measurements and muon imaging technique the cloud movement was tracked which allowed approximate estimates of linear and angular velocity, height and area covered by the thundercloud. Apart from these, electrical properties like potential, capacitance, charge, energy, power delivered by the cloud were also measured. This study suggests that electric potential of $>1.3 \mathrm{GV}$ is required to produce large change in $\Delta \mathrm{I}_{\mu}$ measured by G3MT. The present estimate is an order of magnitude higher than direct measurements available till date which is $0.13 \mathrm{GV}$ [2]. The electric potential of the thundercloud can be measured using balloon soundings by initiating artificial lightnings. These measurements are mainly limited by factors of cloud coverage both on horizontal and vertical planes and duty cycle. The fact that detection of $100 \mathrm{MeV} \gamma$-rays by AGILE satellite implies the necessity of Giga-Volt potential required to produce very high energy electrons for bremsstrahlung of high energy $\gamma$-rays [3]. Also the development of Giga-Volt potential in thunderclouds was first predicted by C.T.R. Wilson 90 years ago [4].

The potential estimation by GRAPES-3 was derived with aid of monte carlo simulations using CORSIKA [5] which itself relies on external hadronic interaction models categorised broadly into low and high energy. Hadronic interaction models are built on theoretical ground with different assumptions. They are calibrated based on available data from collider experiments and extrapolated for entire span of energy spectrum. The number of secondaries given by CORSIKA and its properties vary between models for same shower due to different theoretical approaches and selection effects on first and subsequent interactions in the atmosphere during shower development. Hence the electrical properties derived from simulations may vary on choice of models used. Though this effect was briefed in the report [1], the detailed variations of those models are discussed in this work.

\section{The GRAPES-3 Muon Telescope}

The G3MT is an integral part of GRAPES-3 extensive air shower experiment primarily used to (i) discriminate hadron initiated showers for $\gamma$-ray astronomy, (ii) study primary cosmic rays (PCR) of energy ranging from few $\mathrm{TeV}$ to few tens of $\mathrm{PeV}$ and (iii) measure angular flux of muons for atmospheric and space weather studies [6]. Proportional counter (PRC) is a basic detection element of G3MT. It is made up of mild steel tube with dimension of $10 \mathrm{~cm} \times 10 \mathrm{~cm} \times 600 \mathrm{~cm}$ and wall thickness of $2.3 \mathrm{~mm}$. A muon telescope module consists four layers of PRCs. Each layer embodies 58 PRCs in a row, and alternative layers are placed orthogonal to each other. The layers are sandwiched by $15 \mathrm{~cm}$ thick concrete. On top of all four layers, $2 \mathrm{~m}$ thick concrete is placed in the shape of an inverted pyramid. The total mass overburden of $550 \mathrm{~g} \mathrm{~cm}^{-1}$ ensures $1 \mathrm{GeV}$ threshold for vertical muons and $\sec (\theta) \mathrm{GeV}$ for muons incident at an angle $\theta$. The four layer configuration allows muon reconstruction in two mutually perpendicular planes and two PRC layers in same 


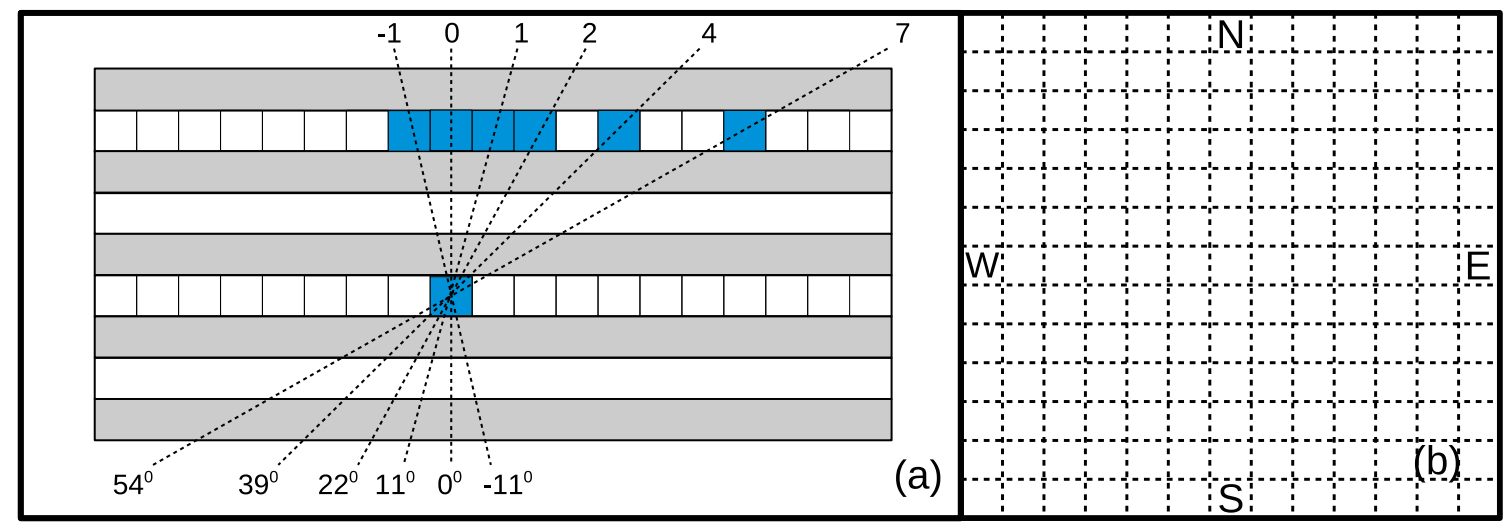

Figure 1: (a) Reconstruction of muon direction in one projection, (b) Reconstructed muons binned into $13 \times 13=169$ direction sky map

projection plane separated by $\sim 50 \mathrm{~cm}$ permit muon direction to be measured with $\sim 4^{\circ}$ accuracy as shown in Fig. 1a. There are 16 muon telescope modules form the G3MT with total area of $560 \mathrm{~m}^{2}$. The muon directions can be reconstructed into 169 directions with field of view of $2.3 \mathrm{sr}$ as shown in Fig. 1b. The G3MT records 4 billion muons everyday which allows $\Delta \mathrm{I}_{\mu}$ to be measured with an accuracy of $0.002 \%$.

\section{Hadronic Interaction Models}

CORSIKA v74001 is used for this study. As mentioned in previous section there are several hadronic interaction models are currently interfaced with CORSIKA which allows user to select a combination of low (interactions $<80 \mathrm{GeV}$ ) and high energy (interactions $>80 \mathrm{GeV}$ ). The list of interfaced models include FLUKA 2011-2B [7] (hereafter FLUKA), GHEISHA 2002d [8] (hereafter GHEISHA) and UrQMD 1.3 cr [9] (hereafter UrQMD) for low and DPMJET 2.55 [10], EPOS LHC [11] (hereafter EPOS-LHC), NEXUS 3.97 [12], QGSJET 01C [13], QGSJETII-04[14] (hereafter QGSJETII), SIBYLL 2.1 [15] (hereafter SIBYLL) and VENUS 4.12 [16] for high energy interactions. Since the muons recorded by G3MT are predominantly produced by PCR of energy $10 \mathrm{GeV}-10 \mathrm{TeV}$, low energy hadronic interactions play major role in muon production than high energy. Hence all three low energy models are used. But only three well known models namely SIBYLL, EPOS-LHC and QGSJETII are used for high energy interactions. Also these high energy models intrinsically use models DPMJET, VENUS and NEXUS. Brief description on theoretical front of these models are described below.

\subsection{FLUKA}

FLUKA is a general purpose tool developed for calculation of particle transport and interactions with matter, covering an extended range of applications. It has an inbuilt package to handle complex geometries, but not used in CORSIKA. It can simulate the interaction and propagation of many particles including photons, electrons, neutrinos, muons and hadrons with high accuracy. Each category of particles are accurate in certain energy ranges. The hadron-nucleon interactions 
are based on resonance production and decay below few $\mathrm{GeV}$ and Dual Parton model (DPM) for higher energies. However, nucleus-nucleus interactions are treated through external event generators like DPMJET, RQMD and BME for various energy ranges. Transport of charged hadrons and muons are treated based on multiple Coulomb scattering and ionisation fluctuations. The treatment of electrons are achieved with original transport algorithm for charged particles. And pair production with actual angular distribution of electrons and positrons is used for photons. This is one of the actively maintained models with frequent updates to give improvements [7].

\subsection{GHEISHA}

The GHEISHA (Gamma-Hadron-Electron Interaction SH(A)ower) code describes the development of a hadronic and/or electromagnetic shower within the materials of experimental setup. Tracking involves multiple scattering and energy loss. The treatment of interactions, nuclear excitation, fermi-motion and generation of secondary particles in nuclear cascades is strongly based on experimental results [8].

\subsection{UrQMD}

The Ultra-relativistic Quantum Molecular Dynamics (UrQMD) is a microscopic model for proton-proton, proton-nucleus and heavy ion collisions at relativistic energies. The transport approach is based on covariant propagation of color strings, constituent quarks and diquarks (as string ends) accompanied by mesonic and baryonic degrees of freedom. It simulates multiple interactions of in-going and newly produced particles, the excitation and fragmentation of colour strings and the formation and decay of hadronic resonances [9].

\subsection{SIBYLL}

SIBYLL is a relatively simple model intended for air shower simulations based on DPM, the Lund monte carlo algorithms and minijet model. The hard interaction cross section is calculated according to minijet model. For hadron-nucleus interactions, the interaction probability for each nucleon inside the nucleus is calculated based on the impact parameter distribution. The total interaction cross section is calculated using the Glauber scattering theory. For a nucleus-nucleus interaction the semi-superposition model is used to determine the point of first interaction for the nucleons of the projectile nucleus. It is optimised to reproduce many features of hadronic interactions in fixed target and collider experiments [15].

\subsection{EPOS-LHC}

EPOS-LHC is successor of EPOS 1.99 which was calibrated to reproduce various experiments of LHC data released since 2009. The data sets include proton-proton, proton-lead and lead-lead interactions. EPOS is based on the Parton-Based Gribov Regge Theory developed for NEXUS, which was based on the VENUS model for soft interactions and the QGSJET model for the semihard scattering. It accounts for many elementary collisions including nucleus-nucleus scattering, proton-proton scattering, known as "parton ladder" or pomeron [11]. 


\subsection{QGSJETII}

The primary motivation behind the original QGSJET model was that there is no principal difference between soft and hard processes in hadron scattering. In order to develop a unified scheme, both soft and semi-hard parton cascades have been treated in QGSJET and later, in the NEXUS model in the framework of Gribov Regge Theory, describing them as exchanges of "soft" and "semi-hard" pomerons. The improvements in this model mainly EAS fluctuations are implemented and strongly constrained by the data from TOTEM experiment of LHC which is mainly by proton-proton cross sections. Another notable improvement is treatment of charge exchange processes in pion-proton and pion-nucleus collisions [14].

\section{CORSIKA Simulations}

Since composition of PCR is dominated by proton $(\sim 90 \%)$, the simulations were carried out for proton primaries in the energy range of $10 \mathrm{GeV}-10 \mathrm{TeV}$. The primaries were simulated efficiently based on geomagnetic cutoff rigidity by a special treatment in CORSIKA [17]. For thunderstorm event recorded on 1 December 2014, the $\Delta \mathrm{I}_{\mu}$ was measured on minute time-scale $\left(\sim 2.5 \times 10^{6} \mathrm{~min}^{-1}\right)$ with the precision of $0.1 \%$. However, due to atmospheric effects and zenith angle dependence, the muon flux drops from vertical to extreme inclined direction by a large factor which results in error ranging $0.4-2.7 \%$. To compensate this varying statistical errors of directional muon flux, instead of using an usual angular distribution for primaries, the simulations were carried out for each of 169 directions by targeting the primaries to center of the direction. Also the number of primaries to be simulated was scaled appropriately to produce $10^{5}$ muons $(0.3 \%$ error $)$ in each of 169 directions for every step of applied electric potential (V). This schema was used to simulate response of $\Delta \mathrm{I}_{\mu}$ for $\mathbf{V}$ in the range of -3 to $3 \mathrm{GV}$ in steps of $0.5 \mathrm{GV}$ at the altitude of $8-10 \mathrm{~km}$ amsl using a approximated cloud model implemented in CORSIKA. The technical details of cloud model implementation is described elsewhere [1, 18]. The response of $\Delta \mathrm{I}_{\mu}$ as a function of $\mathbf{V}$ was derived for all 169 directions after inclusion of detector geometry, threshold and trigger criteria. This exercise was carried for nine combinations from low (FLUKA, GHEISHA and UrQMD) and high (SIBYLL, EPOS-LHC, QGSJETII) energy hadronic interaction models.

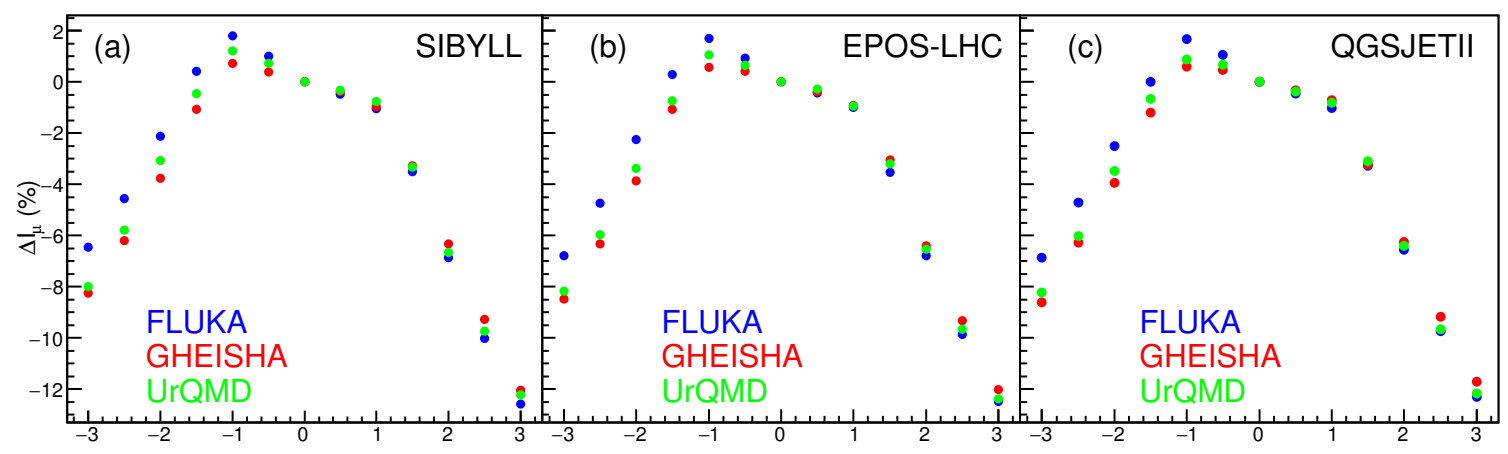

Figure 2: Dependence of $\Delta \mathrm{I}_{\mu}$ derived for electric potential (GV) showing variation between low energy models for a selected high energy of (a) SIBYLL, (b) EPOS-LHC and (c) QGSJETII 


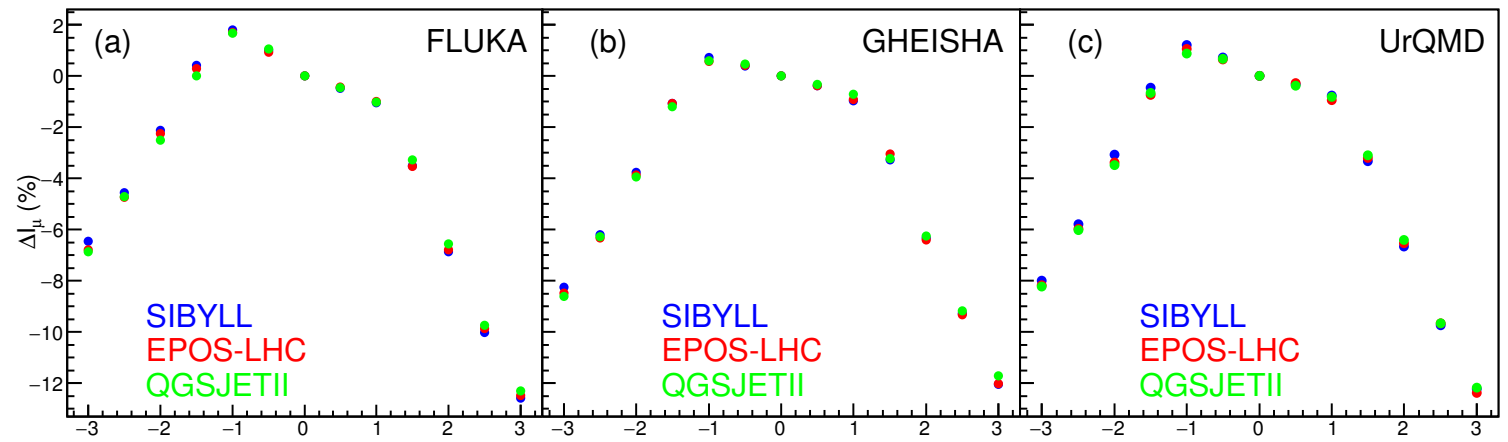

Figure 3: Dependence of $\Delta \mathrm{I}_{\mu}$ derived for electric potential (GV) showing variation between high energy models for a selected low energy of (a) FLUKA, (b) GHEISHA and (c) UrQMD

The simulated profiles of all directions for nine combinations are shown in Fig. 2\&3. The variation of low energy models with respect to a common high energy model is shown in Fig. 2 and vice versa in Fig. 3. These profiles can be used to derive $\mathbf{V}$ required to cause change $\Delta \mathrm{I}_{\mu}$ in G3MT for every direction. From these figures, two broad conclusions can be drawn, (i) $\Delta I_{\mu}$ decreases for $+\mathbf{V}$, and increases for $-\mathbf{V}$ up to $|\mathbf{V}|=\sim 1 \mathrm{GV}$ due to muon charge asymmetry $\left(\mu^{+} / \mu^{-}>1\right)$ found in nature, (ii) however, $\Delta \mathrm{I}_{\mu}$ decreases rapidly beyond certain value due to increased decay probability of muons irrespective of polarity of the $\mathbf{V}[1,18]$.

\section{Results and Discussions}

Aiming to extract the hadronic interaction model dependence, maximum decrease in $\Delta \mathrm{I}_{\mu}(-$ $2 \%$ ) recorded by thunderstorm event on 1 December 2014 (Fig. 3 in [1]) is used to get $\mathbf{V}$ for 45 selected directions (Fig. 2 in [1]) from simulated profiles of nine combinations. The profiles of these combinations are modelled by higher order polynomial to estimate the value of $\mathbf{V}$ and their differences with respect to SIBYLL-FLUKA combination are shown in Table. 1. It is to be noted that SIBYLL-FLUKA combination gives the lowest estimate among these models. Hence the measured electric potential and other properties of the thundercloud are the most conservative estimates using SIBYLL-FLUKA combination as quoted here [1]. Largest estimate is given by

\begin{tabular}{|l|l|l|l|}
\hline & FLUKA & GHEISHA & UrQMD \\
\hline SIBYLL & 0.89 & 1.07 & 1.00 \\
& -- & $(20 \%)$ & $(12 \%)$ \\
\hline EPOS-LHC & 0.93 & 1.07 & 0.97 \\
& $(4 \%)$ & $(20 \%)$ & $(9 \%)$ \\
\hline QGSJETII & 0.96 & 1.12 & 1.03 \\
& $(8 \%)$ & $(26 \%)$ & $(16 \%)$ \\
\hline
\end{tabular}

Table 1: Table consists of $\mathbf{V}(\mathrm{GV})$ required to cause $\Delta \mathrm{I}_{\mu}=-2 \%$ change for thunderstorm event 1 December 2014. Columns and rows represent low and high energy hadronic interaction models respectively and values within parentheses show percentage difference with respect to SIBYLL-FLUKA combination. 
QGSJETII-GHEISHA combination. As discussed in previous section, the difference between high energy models is much smaller compared to the difference between low energy models. However, the quoted differences are not constant, it may increase significantly beyond $1 \mathrm{GV}$ due to change in slope as seen in Fig. 2\&3. Also it can be seen in Fig. 2 that the differences are very large between low energy models for $\mathbf{- V}$ compared to $\mathbf{V}$. These differences are mainly due to the intrinsic properties of models which result in different muon charge asymmetry and decay probability. The differences estimated in $\mathbf{V}$ may result significant increase in other parameters like capacitance, charge, energy and power. After the release of LHC collision data at $14 \mathrm{TeV}$, these models are recalibrated using post-LHC data. The updated models are shown to have better agreement among themselves compared to pre-LHC models [19]. However, post-LHC models may have less impact on electrical properties due to the fact that G3MT's muon measurements mainly rely on low energy hadronic interaction models, but this is yet to be confirmed.

\section{Acknowledgments}

D.B. Arjunan, V. Jeyakumar, S. Kingston, K. Manjunath, S. Murugapandian, S. Pandurangan, B. Rajesh, K. Ramadass, V. Santhoshkumar, M.S. Shareef, C. Shobana, R. Sureshkumar are thanked for assistance in running the GRAPES-3 experiment. We thank SERB-DST of government of India and ICRC organizers for providing partial support to attend this conference.

\section{References}

[1] B. Hariharan et al., Phys. Rev. Lett. 122, 105101 (2019).

[2] T.C. Marshall and M. Stolzenburg, J. Geophys. Res. 106, 4757 (2001).

[3] M. Tavani et al., Phys. Rev. Lett. 106, 018501 (2011).

[4] C.T.R. Wilson, Nucl. J. Franklin Inst. 208, 1 (1929);

Proc. Phys. Soc. London 37, 32D (1924);

Proc. R. Soc. A 236, 297 (1956).

[5] https://www.ikp.kit.edu/corsika

[6] S.K. Gupta et al. Nucl. Instrum. Methods A 540, 311 (2005);

Y. Hayashi et al. Nucl. Instrum. Methods A 545, 643 (2005).

[7] http://www.fluka.org/references.html

[8] http://cds.cern.ch/record/162911/files/CM-P00055931.pdf

[9] http://urqmd.org

[10] J. Ranft, Phys. Rev. D51 (1995) 64; arXiv:hep-ph/9911213 and hep-ph/9911232 (1999).

[11] T. Pierog et al., arXiv:1306.0121[hep-ph] (2013).

[12] H.J. Drescher, M. Hladik, S. Ostapchenko, T. Pierog, and K. Werner, Phys. Rep. 350 (2001) 93Âă(preprint hep-ph/0007198 (2000)).

[13] N.N. Kalmykov, S.S. Ostapchenko, and A.I. Pavlov, Nucl. Phys. B Proc. Suppl. 52B, 17 (1997). 
[14] S.S. Ostapchenko, Phys. Rev. D83 (2011) 014018.

[15] E.J. Ahn, R. Engel, T.K. Gaisser, P. Lipari, and T. Stanev, Phys. Rev. D 80, 094003 (2009).

[16] K. Werner, Phys. Rep. 232 (1993) 87.

[17] B. Hariharan et al., Proceedings of Science PoS(ICRC2015)448.

[18] B. Hariharan et al., Proceedings of Science PoS(ICRC2017)305.

[19] T. Pierog et al., Proceedings of Science PoS(ICRC2017)1100. 